\title{
Internal kinematics of the TW Hya association of young stars
}

\author{
V. V. Makarov and C. Fabricius \\ Copenhagen University Observatory, Juliane Maries Vej 30, 2100 Copenhagen $\varnothing$, Denmark
}

Received 28 June 2000 / Accepted 5 January 2001

\begin{abstract}
Thirty one probable kinematic members of the nearby TW Hya association of young stars are selected from the RASSBSC/Tycho-2 sample of stars luminous in X-rays, detected by ROSAT. Eight of them have been listed already as members of the association, and 23 are new candidates. The association occupies a volume of some $10^{6} \mathrm{pc}^{3}$, the nearest member being at a distance of only $17.5 \mathrm{pc}$ from the Sun. The lower bound internal velocity dispersion is estimated at $0.8 \mathrm{~km} \mathrm{~s}^{-1}$, which is considerably larger than is expected in a gravitationally bound open cluster. The total mass of the group is estimated at 31 solar masses. The centre of mass lies at $73 \mathrm{pc}$ from us in the direction close to the position of the prototype star TW Hya. A realistic kinematical model fitting both the observed trigonometric parallaxes and radial velocities involves a linear expansion of the group with the rate $0.12 \mathrm{~km} \mathrm{~s}^{-1} \mathrm{pc}^{-1}$, which defines a dynamical age of $8.3 \mathrm{Myr}$, in good agreement with previous age estimations for $\mathrm{T}$ Tauri members. Likewise the nearby Carina-Vela moving group of X-ray stars, the TW Hya association appears to be an outpost of the Gould Belt structure rather than an isolated open cluster.
\end{abstract}

Key words. open clusters and associations: individual: TW Hya - Stars: kinematics - X-rays: stars

\section{Introduction}

The TW Hya association (TWA), with the prototype star TW Hya $=$ HIP 53911 at a distance $56 \pm 7$ pc from the Sun, is one of the nearest groups of young co-moving stars. The origin of such groups remains mysterious. They are often not associated with any conspicuous clouds of interstellar matter that could sustain star formation, despite their young age of order $10 \mathrm{Myr}$. The implication is that either the progenitor molecular cloud was somehow effectively dispersed, or decelerated by interaction with intercloud medium (Jones \& Herbig 1979). A small group of stars like the TWA could conceivably be born in a small compact cloudlet which by itself could be an escaper from a larger turbulent cloud (Feigelson 1996).

The present census of the TWA includes at least 13 star systems (Webb et al. 1999; Sterzik et al. 1999; Webb \& Zuckerman 2000), all exhibiting enhanced X-ray luminosities and spectra typical of $\mathrm{T}$ Tauri stars. Their common origin is further confirmed by similar kinematics, i.e. radial velocities and proper motions.

The new data used in this paper are accurate proper motions from the Tycho-2 catalogue (Høg et al. 2000a) for bright X-ray stars observed by ROSAT. The original sample of ROSAT sources identified with Tycho-2 stars is described in Sect. 2. Inspired by the young moving group in Carina-Vela (Makarov \& Urban 2000), which seems to

Send offprint requests to: V. V. Makarov, e-mail: makarov@astro.ku.dk stretch over $100 \mathrm{pc}$ in the radial direction and across the sky, we extend the search area and select 23 new kinematic members of the association in addition to 8 previously known. The selection is described in Sect. 4. The extended list of candidate members permits us to construct a kinematic model of the group, which is a development of the classical convergent point method, as described in Sect. 3. Finally, Sect. 5 (Conclusions) concerns some implications of the kinematic model and the issue of a possible relation between the TWA and the Gould Belt.

\section{The RASSBSC/Tycho2 sample}

The Tycho-2 Catalogue contains accurate positions, proper motions and two-colour photometry for 2.5 million stars. Magnitudes in two passbands $B_{\mathrm{T}}$ and $V_{\mathrm{T}}$ are given for all the stars in the catalogue, and accurate proper motions for $96 \%$ of them. The errors of $V_{\mathrm{T}}$ magnitudes are within $0.05 \mathrm{mag}$ at $V_{\mathrm{T}}=10 \mathrm{mag}$ and within $0.16 \mathrm{mag}$ at $11.5 \mathrm{mag}$. The median standard error of proper motions is $2.5 \mathrm{mas} / \mathrm{yr}$ at $V_{\mathrm{T}}=11.5 \mathrm{mag}$ and $1.5 \mathrm{mas} / \mathrm{yr}$ at magnitude 9.5. It is presently the most accurate reference catalogue among those statistically complete down to $V=11.5$ mag.

The proper motions in Tycho-2 were computed by combining the Tycho- 2 positions at epoch about 1991.5 with those from the Astrographic Catalogue at epoch about 1905 and 143 other transit circle and photographic catalogues, generally at intermediate epochs. Systematically, the proper motions reproduce 
the Hipparcos reference system extremely well (Urban et al. 2000). With regard to accidental errors, they are quite similar in quality to Hipparcos proper motions, but they are more accurate in case of binary stars, being less perturbed by orbital motions with periods much below 100 yr (Makarov et al. 2000).

Another major source of information in this paper is the RASS Bright Source Catalogue, based on the observations in the $0.1-2.4 \mathrm{keV}$ energy band obtained with the PSPC instrument of the ROSAT satellite (Voges et al. 1999). It includes 18811 sources down to a limiting PSPC count rate of $0.05 \mathrm{cts} \mathrm{s}^{-1}$. These data represent the most complete and sensitive X-ray sky survey ever published.

The RASSBSC/Tycho-2 sample was obtained by positional cross-identification of sources in the RASS BSC and Tycho-2 catalogues. Two objects were reckoned matching in position if the angular distance between them was smaller than $3 \sigma_{\text {RASS }}$, where $\sigma_{\text {RASS }}$ is the RASS BSC positional error, which is generally a few tens of arcsec. The Tycho-2 Catalogue contains double stars resolved down to separation 0.8 arcsec. Some of the X-ray sources were therefore matched to 2 or even 3 Tycho- 2 stars. Since there is no way to reasonably tell which of the optical counterparts is responsible for the X-ray emission, all such double identifications, constituting about $10 \%$ of the total, were retained. The sample contains 7096 Tycho-2 stars matched to 6594 RASS BSC sources.

\section{The convergent point method}

Following A. Heck (1978), two closely related methods to infer distances of members of a cluster are distinguished, the convergent point method and the moving cluster method. For a comprehensive historical overview of the methods the reader is referred to Dravins et al. (1999). The methods arise, conceptually, from the same principle. If a group of stars of a sizeable angular extent share the same motion in space, with respect to the observer, then, upon the geometrical projection onto the celestial sphere, the angular extent will change with time, and the cluster will contract, in infinity, to a single point. Translated into observed proper motions, the geometrical projection effect results in a measurable gradient of proper motion components across the cluster (the moving cluster method), or into a common convergent point, in which all the proper motion vectors intersect when extended around the sphere (the convergent point method). Rather arbitrarily, these two methods may be distinguished by whether the convergent point coordinates are explicitly estimated from the directions of proper motion vectors. Since coordinates of the convergent point are explicitly determined in this paper, the method in use is a variant of the convergent point method.

The general recipe of the method is:

1) Determine coordinates of the convergent point from the available proper motion directions; 2a) Determine the centroid velocity vector from the convergent point, proper motion magnitudes and available radial velocities, or

2b) Determine the centroid velocity vector from the convergent point, proper motion magnitudes and available trigonometric parallaxes;

3) Determine kinematic parallax and radial velocity for each star.

Traditionally, the option 2a) was used by many authors, because trigonometric parallaxes were too scarce and imprecise in comparison with radial velocities. The advance of the Hipparcos Catalogue in 1997 changed the situation, and now radial velocity data often lag in quality and number. Lindegren et al. (2000), in their all-round maximumlikelihood approach use Hipparcos parallaxes as the original data to derive astrometric radial velocities. We do the same in this paper. We demonstrate, furthermore, that a comparison of astrometric radial velocities with observed radial velocities can reveal the presence of internal expansion or contraction in a cluster, and we develop the classical convergent point method to accomodate an isotropic uniform expansion.

Following the designations in (Makarov et al. 2000), the basic parameters involved in the computation are

$-\Delta$, the angle between the direction of the proper motion for each star and the direction from the star position to the common convergent point of the cluster;

$-\lambda$, the angular distance between the star position and the convergent point;

$-\mu$, the magnitude of proper motion in $\operatorname{arcsec} \mathrm{yr}^{-1}$;

$-v_{\perp}$, the transverse velocity component for each star, i.e. $v \sin \lambda \sin \Delta$;

- $\sigma_{\mu \perp}$, the formal standard error of the transverse proper motion component normal to the convergent point direction, which is computed from the coordinate proper motion components $\mu_{\alpha} \cos \delta=\mu_{\alpha *}$ and $\mu_{\delta}$;

$-\boldsymbol{v}_{\mathrm{C}}$, the centroid velocity of the group;

- $\sigma_{v}$, the internal dispersion of velocities, assumed to be isotropic within the group.

An important quantity reflecting the degree of proper motion convergence for each star, is the relative directional deviation of the proper motion vector, $\Delta / \sigma_{\Delta}$, where $\sigma_{\Delta}$ is the total expected standard error of $\Delta$. The error is a quadratic sum of the relative astrometric error and the relative tangential velocity dispersion

$\sigma_{\Delta}^{2}=\frac{\sigma_{\mu \perp}^{2}}{\mu^{2}}+\frac{\sigma_{v}^{2}}{v_{\mathrm{C}}^{2} \sin ^{2} \lambda}$

The first step of the procedure is to estimate the convergent point coordinates. Using the Tycho-2 proper motions only, convergent point coordinates are solved for by a $\chi^{2}$ adjustment, i.e., the point is determined which yields the minimum quadratic sum of $\Delta / \sigma_{\Delta}$ over the selected stars. The actual adjustment is iterative, because apart from the coordinates one has to estimate $\sigma_{v}$ and $v_{\mathrm{C}}$, needed to compute the residuals $\Delta / \sigma_{\Delta}$. Usually, a few iterations are 
required for the adjustment to converge. Equation (1) allows one to estimate the internal dispersion $\sigma_{v}$, once the convergent point and the bulk velocity $v_{\mathrm{C}}$ are known. The computed distribution of $\Delta / \sigma_{\Delta}$ values, as a function of $\sigma_{v}$, is expected to have a standard deviation of unity. The standard deviation may be estimated by fitting a Gaussian to the observed distribution, but for a small sample like ours the percentile method is more robust. The $\sigma$ is defined as the half difference between the 84th and 16th percentiles over the sample, and it tends to give conservative (overestimated) values for non-Gaussian distributions. Throughout this paper, we estimate $\sigma_{v}$ simply by forcing 32 percent of the sample to have $\left|\Delta / \sigma_{\Delta}\right|>1$.

The next step is estimating the total bulk velocity of the group $v_{\mathrm{C}}$. At this point, we know the angular distance $\lambda$ between each star and the convergent point. Using available trigonometric parallaxes, the centroid velocity is derived from

$v_{\mathrm{C}} \sin \lambda=4.74 \mu \pi^{-1}$.

It was pointed out recently (Dravins et al. 1999), that isotropic uniform expansion or contraction is the only parameter represented by the general velocity field tensor of a cluster, that can not be derived from proper motions and positions only. This singularity can be clearly seen from the geometrical observation, that this kind of systematic motion still makes the projected tangential motions cross in one point. The presence of isotropic expansion, however, results in a discrepancy between the astrometric radial velocities

$v_{r}=v_{\mathrm{C}} \cos \lambda$,

and observed radial velocities $V_{\text {rad }}$. A uniform expansion within a cluster, for example, shifts the apparent convergent point farther from the cluster, and decreases the astrometric radial velocities through increasing $\lambda$. In the classical convergent point method, as described by Heck (1978), the motion of each star is assumed to be the same as the centroid motion

$\boldsymbol{v}=\boldsymbol{v}_{\mathrm{C}}$

We will call it hereafter Model I. Our assumption for a more sophisticated Model II is that each individual velocity vector

$\boldsymbol{v}=\boldsymbol{v}_{\mathrm{C}}+\boldsymbol{v}_{\exp }=\boldsymbol{v}_{\mathrm{C}}+R_{\exp } \boldsymbol{r}_{\mathrm{C}}$,

where $\boldsymbol{r}_{\mathrm{C}}$ is the position vector with respect to the centre of mass and $R_{\text {exp }}$ is the uniform expansion rate. In this model, a new convergent point is determined after correcting all the measured proper motions for the uniform expansion, in an iterative adjustment involving two more parameters, the distance to the cluster centre $D_{\mathrm{C}}$ and $R_{\text {exp }}$.

The centre of mass is determined in the following way. For each star, we convert the observed Tycho- 2 colour $B_{\mathrm{T}}-V_{\mathrm{T}}$ into Johnson $B-V$, and $V_{\mathrm{T}}$ magnitude into $V$, using the approximate relations given in Vol. 1 of ESA

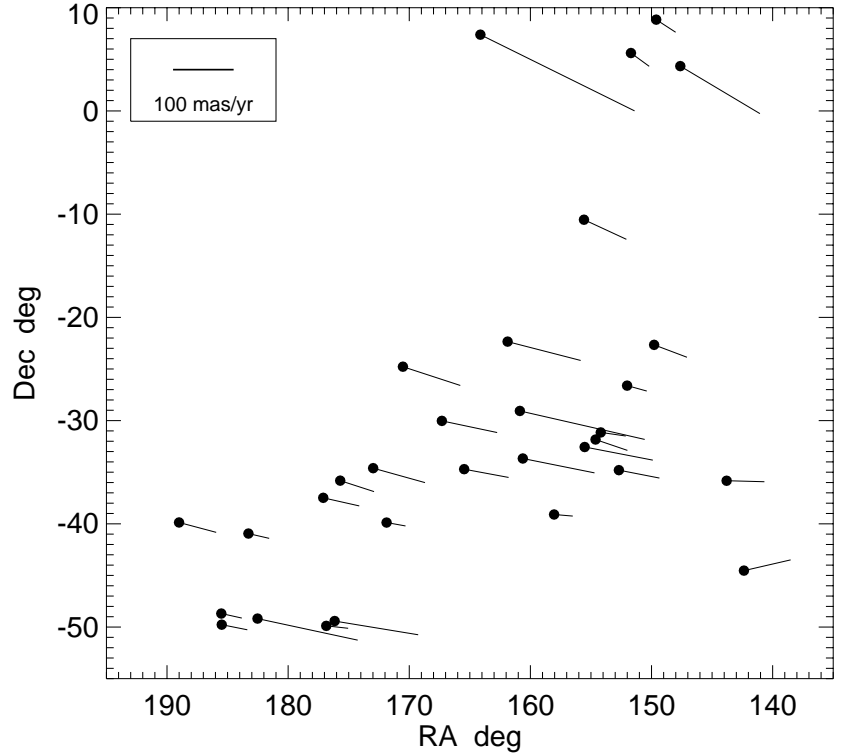

Fig. 1. Positions and proper motions of the 31 selected kinematic members of the TWHya association

1997. Absolute magnitudes $M_{V}$ are computed from the estimated kinematic distances. Using the formula in Reid (1992) for main sequence stars, the mass is estimated from the absolute magnitude and colour. Finally, the masses, positions and kinematic distances are used to determine the centre of mass location. In the basic kinematic Model I, this procedure is straightforward, because the kinematic distances are independent of the centre of mass location. In the second model, with non-zero expansion rate, the process becomes iterative, since the model velocity for each star, and hence the kinematic distance, depends on the centre of mass location.

\section{Kinematics and membership of the TW Hya association}

\subsection{Selection of new members}

We inspect all RASSBSC/Tycho-2 stars with measured proper motions in a large area of the sky of above $3000 \mathrm{deg}^{2}$ depicted in Fig. 1. The stars are selected with proper motions greater than 30 mas/yr, crossing a circle of radius $5^{\circ}$ centred on $(l, b)=\left(222^{\circ} .3,-27^{\circ} .9\right)$ which is the convergent point of the "classical" members, determined by a non-linear $\chi^{2}$ adjustment of Model I. The cut on $\mu$ is meant to clean the sample of more distant kinematic interlopers, but it may in fact leave out real distant members of TWA. The limit of $5^{\circ}$ may seem restrictive, because two previously known members of the association, TWA 6 and 11, deviate by more than this angle from the assumed convergent point. They would not have been included by our criteria, meaning that probably more loosely associated members could be found in the area, and that the internal velocity dispersion of $0.8 \mathrm{~km} \mathrm{~s}^{-1}$ derived in the next section might be an underestimation. 
Table 1. Kinematic members of the TW Hya association. HIP and TYC identification numbers are given in Col. 1, and internal TWA numbers for known members in Col. 2. The equatorial coordinates are given in the ICRS system (Cols. 3 and 4 ). Magnitudes of proper motions from the Tycho-2 catalogue are given in Col. 5. $V_{\mathrm{T}}$ magnitudes and $\left(B_{\mathrm{T}}-V_{\mathrm{T}}\right)$ colours from Tycho-2 are given in Cols. 6 and 7. Trigonometric distances for Hipparcos stars are given in Col. 8, and estimated kinematic distances in Col. 9. Column 10 contains radial velocities predicted by the convergent point method (Model II), and Col. 11 observed radial velocities from Simbad. A ":" denotes a known binary star, for which radial velocity may be affected by orbital motion. Absolute directional difference $\Delta$ and relative difference $\Delta / \sigma_{\Delta}$ are in Cols. 12 and 13. Ratio of X-ray luminosity to bolometric luminosity is given in Col. 14 .

\begin{tabular}{|c|c|c|c|c|c|c|c|c|c|c|c|c|c|c|}
\hline star & TWA & $\begin{array}{r}\mathrm{RA} \\
\mathrm{h} \quad \mathrm{m}\end{array}$ & & $\begin{array}{l}\text { Dec } \\
0 \quad,\end{array}$ & $\begin{array}{c}\mu \\
\mathrm{mas} / \mathrm{yr}\end{array}$ & $\begin{array}{l}V_{\mathrm{T}} \quad E \\
\text { mag }\end{array}$ & $\begin{array}{c}B_{\mathrm{T}}-V_{\mathrm{T}} \\
\mathrm{mag}\end{array}$ & $\begin{array}{r}D_{\text {tri }} \\
\text { pc }\end{array}$ & $\begin{array}{c}D_{\text {kin }} \\
\text { pc }\end{array}$ & $\begin{array}{c}v_{r} \\
\mathrm{~km} \mathrm{~s}^{-1}\end{array}$ & $\begin{array}{c}V_{\mathrm{rad}} \\
\mathrm{km} \mathrm{s}^{-1}\end{array}$ & $\begin{array}{c}\Delta \\
\operatorname{deg}\end{array}$ & $\Delta / \sigma_{\Delta}$ & $\log \left(\frac{L_{\mathrm{X}}}{L_{\mathrm{bol}}}\right)$ \\
\hline 53911 & 1 & 110151.9 & -34 & 4217.2 & 75.4 & 11.34 & 0.75 & 56.4 & 57.1 & 12.7 & 13.5 & 0.6 & 0.22 & -3.37 \\
\hline 7201-0027-1 & 12 & 110913.8 & -30 & 0139.8 & 92.6 & 11.36 & 2.21 & & 47.1 & 10.6 & & 2.9 & 0.88 & -4.17 \\
\hline 55505 & 4 & 11225.3 & -24 & 4639.5 & 96.8 & 9.24 & 1.40 & 46.7 & 45.7 & 9.1 & & -0.3 & -0.14 & -4.29 \\
\hline $7223-0275-1$ & 15 & 113155.3 & -34 & 3627.5 & 86.7 & 11.72 & 1.69 & & 50.9 & 10.0 & & -1.8 & -0.66 & -3.33 \\
\hline $7183-1477-1$ & 16 & 101828.7 & -31 & 5002.8 & 60.6 & 12.00 & 1.89 & & 67.4 & 16.2 & & -10.8 & -2.91 & -4.05 \\
\hline $7190-2111-1$ & 17 & 104230.1 & -33 & 4016.6 & 125.7 & 11.06 & 1.54 & & 33.6 & 11.0 & & -1.6 & -0.61 & -3.32 \\
\hline 57589 & 9 & 114824.2 & -37 & 2849.2 & 58.1 & 11.32 & 1.64 & 50.3 & 76.3 & 12.6 & & 1.9 & 0.63 & -3.45 \\
\hline 61498 & 11 & 12361.0 & -39 & 5210.2 & 57.4 & 5.77 & 0.02 & 67.1 & 78.1 & 10.5 & & 4.6 & 1.93 & -5.17 \\
\hline 46535 & & 092928.6 & -44 & 3156.5 & 93.5 & 7.03 & 0.54 & 39.7 & 39.5 & 16.0 & 22.1: & 3.8 & 1.39 & -3.98 \\
\hline 47039 & & 093511.8 & -35 & 4925.5 & 73.9 & 6.52 & 0.46 & 63.4 & 50.5 & 16.9 & 12.71 & -0.3 & 0.10 & -5.40 \\
\hline 48273 & & 095030.1 & +04 & 2037.1 & 157.9 & 6.29 & 0.49 & 45.9 & 26.6 & 10.7 & 17.4: & 4.5 & 1.91 & -5.17 \\
\hline 0829-0845-1 & & 095827.2 & +08 & 5043.7 & 38.4 & 10.11 & 0.81 & & 111.7 & 20.4 & & 3.2 & 0.82 & -3.62 \\
\hline 6604-0118-1 & & 09598.4 & -22 & 3934.6 & 63.7 & 10.09 & 1.26 & & 62.8 & 16.5 & & -5.26 & -1.63 & -3.78 \\
\hline 49530 & & 100647.4 & +05 & 3641.4 & 37.0 & 6.29 & 1.08 & 105.9 & 116.4 & 20.7 & 16.7: & -2.4 & -0.99 & -4.63 \\
\hline 6625-1087-1 & & 10083.5 & -26 & 3638.6 & 36.7 & 11.11 & 1.02 & & 110.1 & 21.3 & & -2.8 & -0.55 & -2.97 \\
\hline $7178-14$ & & 101046.7 & -34 & 4753.1 & 75.2 & 11.61 & 0.86 & & 53.4 & 15.2 & & -5.7 & -1.61 & -2.54 \\
\hline 7183-1879-1 & & 101645.4 & -31 & 0844.2 & 45.1 & 11.58 & 1.12 & & 90.3 & 18.9 & & 1.9 & 0.41 & -2.91 \\
\hline 7188-0575-1 & & 10224.5 & -32 & 3327.0 & 123.3 & 10.26 & 1.31 & & 33.2 & 12.2 & & -2.2 & -0.87 & -3.06 \\
\hline 50796 & & 102218.0 & -10 & 3215.5 & 79.1 & 11.14 & 1.34 & 34.0 & 53.8 & 13.1 & & -0.5 & -0.18 & -2.61 \\
\hline $7710-2231-1$ & & $1032 \quad 10.2$ & -39 & 0546.5 & 32.7 & 9.27 & 1.25 & & 126.4 & 22.5 & & 0.1 & 0.04 & -3.82 \\
\hline 52462 & & 104328.3 & -29 & 0351.4 & 218.3 & 7.81 & 1.02 & 21.6 & 19.4 & 9.0 & & 0.4 & 0.16 & -3.98 \\
\hline 52787 & & 104731.2 & -22 & 2052.9 & 126.5 & 8.44 & 0.96 & 34.9 & 34.1 & 9.9 & & 3.5 & 1.51 & -3.86 \\
\hline 53486 & & 105630.8 & +07 & 2318.5 & 268.1 & 7.47 & 1.09 & 17.6 & 16.7 & 3.7 & & 3.9 & 1.79 & -4.55 \\
\hline 55899 & & 112729.4 & -39 & 5235.4 & 31.2 & 7.33 & 0.08 & 142.5 & 140.3 & 21.8 & & 0.9 & 0.28 & -4.56 \\
\hline 57129 & & 114248.1 & -35 & 4857.5 & 57.0 & 9.37 & 0.64 & 105.2 & 77.7 & 12.9 & 29.1: & -4.0 & -1.56 & -3.48 \\
\hline 57269 & & 114438.5 & -49 & 2502.7 & 135.9 & 9.12 & 1.02 & 48.6 & 32.2 & 8.5 & & -1.2 & -0.53 & -2.84 \\
\hline 57524 & & 114724.5 & -49 & 5303.0 & 34.9 & 9.14 & 0.70 & 104.0 & 125.5 & 20.0 & & 2.4 & 0.87 & -3.52 \\
\hline 59315 & & 12106.5 & -49 & 1050.7 & 160.3 & 8.24 & 0.78 & 37.8 & 27.7 & 6.6 & & 0.0 & 0.01 & -4.17 \\
\hline $7760-0835-1$ & & 12137.0 & -40 & 5631.6 & 32.7 & 9.86 & 0.60 & & 136.5 & 19.4 & & 3.3 & 0.99 & -3.55 \\
\hline 8238-1462-1 & & 122155.7 & -49 & 4612.5 & 40.0 & 10.10 & 0.90 & & 111.1 & 16.8 & & 2.8 & 1.00 & -3.85 \\
\hline 8234-2856-1 & & 12224.3 & -48 & 4125.0 & 32.4 & 10.59 & 0.95 & & 137.6 & 20.1 & & 1.8 & 0.48 & -3.31 \\
\hline
\end{tabular}

This selection yields 31 stars, including 8 previously known members adopted from the literature. They are listed in Table 1. Using X-ray fluxes measured by ROSAT, the $V_{\mathrm{T}}$ and $B_{\mathrm{T}}$ magnitudes from the Tycho-2 Catalogue, and tabulated bolometric corrections for main sequence stars from Johnson (1966), the ratio of X-ray to bolometric luminosities are computed (last column in Table 1). The Tycho magnitudes and colours are transformed into the Johnson system by the approximate relations for main sequence stars given in (ESA 1997, Vol. 1). It is noted that our $\log \left(L_{\mathrm{X}} / L_{\mathrm{bol}}\right)$ values are systematically smaller than those given by Webb et al. (1999). The reason for this discrepancy is difficult to find out, because Webb et al. do not describe explicitly which bolometric correction they have used. Our estimates vary from -5.4 for HIP 47039 and -5.2 for HIP 48273 to -2.5 for TYC $7178-1493-1$. The X-ray performance of the former two would be modest even by the 600 Myr old Hyades standards (Stern et al. 1995), although such faint sources are indeed observed sometimes in the much younger Pleiades (Micela et al. 1999) and $\alpha$ Per (Randish et al. 1996) clusters. The star HIP 47039 has a measured radial velocity of $+12.71 \pm 0.27$, not too different from the predicted +16.9 (Table 1). The star HIP $48273=$ HD 85217 is a spectroscopic binary, and therefore its observed radial velocity of +17.4 may be disturbed, against the predicted +10.7 . But a short-period orbital motion could hardly hamper so much the Tycho- 2 proper motions. It seems 
more likely that the velocity of this star is about $42 \mathrm{~km} \mathrm{~s}^{-1}$ instead of the $25 \mathrm{~km} \mathrm{~s}^{-1}$ as estimated for the association. Summarizing, these two stars are quite likely statistical interlopers in our sample.

Another relatively moderate X-ray emitter in the sample is HIP $53486=$ HD 94765. It is potentially the nearest member of the association. Spectroscopic observations are needed to establish its age and to determine its radial velocity. The predicted radial velocity in the kinematic model II is low $(+3.7)$, because it is dumped by the counter expansion velocity component (see next section). Whether the true $V_{\text {rad }}$ is so low should provide a check on our assumption of a linear expansion out to distances above $50 \mathrm{pc}$ from the centre.

The HIP $46535=$ HD 82241 is related by Eggen (1995) to pre-main-sequence stars in the Pleiades supercluster. The known radial velocity of it $(+22.1$ in the Simbad data base) is not in a good agreement with the predicted +16.0 . That this star is an interloper can not be precluded.

Observational evidence of young age have been collected and published for some of the new additions to the TWA list, apart from the high X-ray activity. The HIP $59315(\mathrm{G} 5 \mathrm{~V})$ is known to be chromospherically active. The TYC 6604-0118-1 = SAO $178272(\mathrm{~K} 2 \mathrm{~V})$ has a lithium $6707.8 \AA$ equivalent width of $0.143 \AA$ and rotation velocity $v \sin (i)=19 \mathrm{kms}^{-1}$ (Favata et al. 1995), as expected of a young dwarf.

The star HIP $49530=\mathrm{HR} 3973$, one of the most distant in the group, is remarkable by its quoted spectral type K1III. Some K giants, prominent on the X-ray sky, are indeed embedded in the Gould Belt. A young age of this star can not be precluded, although its X-ray luminosity might be ascribed to the binary component. Importantly, two other distant stars, HIP 55899 (A0V) and HIP 57524 (G3/G5p) are related by de Zeeuw et al (1999) to the Lower Centaurus Crux (LCC) OB association, which overlaps with the lower left corner of the area in Fig. 1. Obviously, de Zeeuw et al. would have included some more of the stars in our sample as members of LCC, should they not impose a lower limit on distance to its stars. Reversely, we could find more stars belonging kinematically to LCC by loosing our limit on $\mu>30$ mas/yr. This might mean that the TWA on the large scale is not separated clearly from the background LCC association and the Gould Belt as a whole.

\subsection{Binaries}

The companion star fraction (CSF) for main sequence stars, describing the frequency of binary and triple stars, is known to correlate well with the age of a stellar aggregate (e.g., Patience et al. 1998). Over the binary separation range 5 to $50 \mathrm{AU}$, the CSF drops from about 0.40 for $\mathrm{T}$ Tauri stars (several million years old) through 0.30 in the Hyades (600 Myr) to 0.14 for nearby field G dwarfs. We should expect therefore a high frequency of binaries in our sample. Two stars among the 8 original members, TWA 5 and 6 , are suspected to be spectroscopic binaries
(Webb et al. 1999). HIP $57129=$ HD 101799 is a wellknown contact binary. The number of known visual binaries includes TWA 2 (separation $27 \mathrm{AU}$ ), TWA 5 (97 AU), TWA 9 (302 AU) and TWA 11 (513 AU). To this number, we add from the new members HIP 49530, resolved by speckle interferometry (14 AU) (McAlister et al. 1987) and HIP 57269, resolved by Hipparcos at $\rho=0.428$ arcsec (21 AU).

Attempting to detect more visual binaries, we used the original Tycho- 2 observations and the extant double star reduction software (Høg et al. 2000b) for non-Hipparcos members. Normally, due to the poor photon statistics and broad slit response function of the Tycho instrument, resolutions of stars at separations below about $0.6 \operatorname{arcsec}$ and with component magnitudes fainter than 11.5 would be reckoned ambiguous. Fortunately, some of the stars in the present selection happen to be close to the so-called nodes of the scanning law of the satellite, and thus received a number of observations much above the average (120) and well-distributed in position angle. Still, the results given below for three stars should be taken with caution, because the conditions are really challenging the Tycho instrument capabilities.

The star TYC 7190-2111-1 = TWA 7, with 188 Tycho observations, is resolved into two components at angular separation $1.78 \operatorname{arcsec}(60 \mathrm{AU})$ and position angle 313.9 . The estimated magnitudes are $V_{\mathrm{T}}=11.2, B_{\mathrm{T}}-V_{\mathrm{T}}=1.1$ for the primary, and $V_{\mathrm{T}}=12.1, B_{\mathrm{T}}-V_{\mathrm{T}}=1.3$ for the secondary.

The star TYC 7760-0835-1, with 249 Tycho observations, is resolved at angular separation $0.32 \operatorname{arcsec}(44 \mathrm{AU})$ and position angle 31.8 . The estimated magnitudes are $V_{\mathrm{T}}=10.33, B_{\mathrm{T}}-V_{\mathrm{T}}=0.52$ for the primary, and $V_{\mathrm{T}}=11.09, B_{\mathrm{T}}-V_{\mathrm{T}}=0.76$ for the secondary.

The star TYC 8234-2856-1, with 273 Tycho observations, is resolved at angular separation $0.42 \operatorname{arcsec}(58 \mathrm{AU})$ and position angle $68^{\circ} 2$. The estimated magnitudes are $V_{\mathrm{T}}=11.10, B_{\mathrm{T}}-V_{\mathrm{T}}=0.84$ for the primary, and $V_{\mathrm{T}}=11.59, B_{\mathrm{T}}-V_{\mathrm{T}}=0.94$ for the secondary.

Taking into account all separations, the estimated CSF for the group is 0.42 , in good agreement with the assumed youth, and, quite likely, more double stars are to be resolved.

\subsection{Kinematic model}

For the 31 stars listed in Table 1, Model I yields a convergent point at $(\alpha, \delta)=\left(80.9,-20^{\circ} 0\right) \pm(2.2,1.3)$. In a few iterations, we found a $\sigma_{v}=0.9 \mathrm{~km} \mathrm{~s}^{-1}$, which together with the above mentioned convergent point, provides only 10 deviants with $\left|\Delta / \sigma_{\Delta}\right|>1$ among the 31 stars. This matches the expected number of deviants for a normal distribution of errors.

Using the Hipparcos trigonometric parallaxes for the 17 HIP stars in the sample, we calibrate $v_{\mathrm{C}}$. The formal precision of the Hipparcos distances is typically 10 to 15 percent, while the formal precision of kinematic 
distances (from $\sigma_{v}=0.9 \mathrm{~km} \mathrm{~s}^{-1}$ ) is about 4 percent. The result is a total velocity of $22 \mathrm{~km} \mathrm{~s}^{-1}$.

The inadequacy of this basic model (Model I) emerges when one starts to compare the predicted radial velocities $V_{\mathrm{rad}}=v_{\mathrm{C}} \cos \lambda$ with the observed ones. Webb \& Zuckerman (2000) determined a common radial velocity of $+11 \pm 2 \mathrm{~km} \mathrm{~s}^{-1}$ for several very young stars around TW Hya. Sterzik et al. (1999) found a little larger +13.5 for TW Hya. These measures are obviously in disagreement with velocities in the interval +3.2 to +5.8 predicted from Model I for the original members TWA 1, 2, 4 and 5 . We could not reconcile the observed radial velocities with the Tycho- 2 proper motions even when only a few originally known members were involved. In order to reproduce much higher radial velocities, the true convergent point ought to be considerably closer to the TWA location than the spot where the proper motions do cross. It is concluded, that the observed motions of the TWA stars are somewhat diverted from the true direction of the centroid motion, most likely by a superimposed expansion component. Another option would be an outward velocity component independent of the distance, but in such a model the stars would not be co-eval.

The parameters of the centroid in the second, more viable model are: distance $D_{\mathrm{C}}=73 \mathrm{pc}$, position $\left(\alpha_{\mathrm{C}}, \delta_{\mathrm{C}}\right)=\left(165^{\circ} 4,-32.1\right) \pm(2 \circ 4,1.1)$. Thus, the centroid is at $16 \mathrm{pc}$ from the historically first member TW Hya. The total mass of the 31 stars is $30.7 M_{\odot}$. The estimated velocity dispersion $\sigma_{v}=0.8 \mathrm{~km} \mathrm{~s}^{-1}$ and the expansion rate $R_{\exp }=0.12 \mathrm{~km} \mathrm{~s}^{-1} \mathrm{pc}^{-1}$. The centroid velocity vector in the galactic coordinates ${ }^{1}$ is $(U, V, W)=(-12.7,-20.6,-6.2) \mathrm{km} \mathrm{s}^{-1}$. The peculiar motion of TWA is $(-2.7,-15.3,+1.0) \mathrm{km} \mathrm{s}^{-1}$, assuming a peculiar velocity of the Sun of $(10.0,5.3,7.2) \mathrm{km} \mathrm{s}^{-1}$ (Dehnen \& Binney 1998). This implies that the group moves at $16 \mathrm{~km} \mathrm{~s}^{-1}$ almost directly away from the Sun with respect to the local standard of rest, and that it lags behind the local Galactic rotation. Such an outward motion is characteristic of the nearby Sco-Cen OB associations in the 4th Galactic quadrant.

The estimated velocity dispersion of $0.8 \mathrm{~km} \mathrm{~s}^{-1}$ in one component puts the TWA in between gravitationally bound open clusters like the Hyades with $\sigma_{v}=0.2$ to $0.4 \mathrm{~km} \mathrm{~s}^{-1}$ (Gunn et al. 1988; Perryman et al. 1998; Makarov et al. 2000) and the Pleiades with $\sigma_{v}=0.4$ to $0.6 \mathrm{~km} \mathrm{~s}^{-1}$ (Jones 1970; Geffert et al. 1995), and gravitationally unbound OB associations like Scorpius OB2 with $\sigma_{v} \lesssim 1.0$ to $1.5 \mathrm{~km} \mathrm{~s}^{-1}$ (de Bruijne 1999). However, there is a possibility of bias in our estimation, coming from the limit of $5^{\circ}$ in the selection of proper motion candidates (Sect. 4.1). In order to evaluate the size of the bias, we conducted a simple simulation, introducing various $\sigma_{v}$ into Model I for the given sample of stars. Of the 31 stars, none are expected to miss the $5^{\circ}$ circle at $\sigma_{v}=1.3 \mathrm{~km} \mathrm{~s}^{-1}$, only 4 at $1.5,16$ at 1.8 and all at 2.0. The true dispersion is

1 The $U$ axis is directed towards the Galactic centre, and the $V$ axis along the Galactic rotation. therefore unlikely to be in the interval 0.9 to $1.5 \mathrm{~km} \mathrm{~s}^{-1}$, where our sample appears to be complete. But if the true dispersion is in 1.5 to $2.0 \mathrm{~km} \mathrm{~s}^{-1}$, a severe underestimation may occure. If the true dispersion is greater or equal than $2.0 \mathrm{~km} \mathrm{~s}^{-1}$ our selection is meaningless.

The expansion rate of $0.12 \mathrm{~km} \mathrm{~s}^{-1} \mathrm{pc}^{-1}$ defines a dynamic age of $8.3 \mathrm{Myr}$. This agrees remarkably well with the previous estimations for $11 \mathrm{~T}$ Tauri members in (Webb et al. 1999). Nine of their 11 members are located between the $10^{6}$ and $10^{7} \mathrm{yr}$ isochrones (Fig. 3 loc cit), and only two systems appear to be older (TWA 6 and 9). A spread of ages of several Myr can not be precluded.

\section{Conclusions}

The discovery that very young X-ray active late type stars in the Solar vicinity are associated with the Gould Belt (Guillout et al. 1998) changed our conception of this largely mysterious formation. It becomes clear now that the Belt is rather well filled with dim late type stars of young age, so a Gould Disk or Plane would be a better name. The near edge (if there is any) of the ScoCen OB associations in the 4th Galactic quadrant appears to be clumpy, comprising open clusters like IC 2391 and IC 2602 and loose moving groups like the CarinaVela (Makarov \& Urban 2000). It was proposed that the Carina-Vela group is a swirl or an extension of the nearby Lower Centaurus Crux association, reaching very close to the Sun. The TWA may be a similar extension of the LCC, situated to the north of the Carina-Vela group. In our sample of probable kinematic members of TWA, we do not see any clear separation from the LCC in distances. However, the space velocity of LCC is approximately $(U, V, W)=(-12,-13,-7) \mathrm{km} \mathrm{s}^{-1}$ (de Zeeuw et al. 1999), i.e., deviates from our estimate by $\sim 8 \mathrm{~km} \mathrm{~s}^{-1}$ in the $V$ component, that is mostly in the line-of-sight direction. The reason for this difference might be that our uniform expansion kinematic model does not extrapolate to the more distant LCC. Our model predicts a radial velocity of $\sim+21$ for the LCC, while the observed value is around +12 . If uniform expansion is common for young stellar associations in the Gould Belt, its rate may vary among different groups in accordance with their age. The recent determination of the $K$ term of the systematic velocity field, representing uniform isotropic expansion, resulted in a value of $7.1 \pm 1.4 \mathrm{~km} \mathrm{~s}^{-1} \mathrm{kpc}^{-1}$ for Gould Belt stars younger than $30 \mathrm{Myr}$ at distances 100 to $600 \mathrm{pc}$ (Torra et al. 2000). This clearly shows that the Gould Belt is not expanding from a single point, but was rather born as a large structure. Different parts of the structure may therefore have varying kinematics and age. Interestingly, Torra et al.'s Fig. 14 suggests, that the Gould Belt expansion rate continues to grow rapidly with decreasing distance from the Sun, but, unfortunately, no sufficient data is available yet for the close vicinity within 100 pc. If this trend proves to be real, the difference of $8 \mathrm{~km} \mathrm{~s}^{-1}$ in outwards motion between the LCC and TWA may be a part of the general pattern, rather than a peculiarity. 
A small group of young stars like the TWA, could conceivably be born in a small compact cloud generally associated with the much larger Gould Belt structure. At the star formation burst, the cloud exploded, or started to expand internally, so that the new-born stars acquired a spread of outwards motions with respect to the centre of mass. The remaining diffused matter was also involved in the expansion, and it has become so thin by now that it is very difficult to detect. It would be very important to find out if internal expansion is also present in other nearby pre-main-sequence and $\mathrm{OB}$ associations.

A scrutiny of radial velocities of the 31 selected stars should help to weed out remaining interlopers in the sample and to test our assumption of an isotropic uniform expansion. If the rate of expansion turns out to be rather distance-independent, the more distant stars from the centre should be systematically older than the T Tauri members in the close vicinity of TW Hya.

Acknowledgements. This work was supported by the Danish Space Board. We have made use of the Simbad data base operated at the Centre de Données Astronomiques de Strasbourg.

\section{References}

de Bruijne, J. H. J. 1999, MNRAS, 310, 585

Dehnen, W., \& Binney, J. J. 1998, MNRAS, 298, 387

Dravins, D., Lindegren, L., \& Madsen, S. 1999, A\&A, 348, 1040

de Zeeuw, P. T., Hoogerwerf, R., de Bruijne, J. H. J., et al. 1999, AJ, 117, 354

Eggen, O. J. 1995, AJ, 110, 1749

ESA 1997, The Hipparcos Catalogue, ESA-SP 1200

Favata, F., Barbera, M., Micela, G., \& Sciortino, S. 1995, A\&A, 295, 147

Feigelson, E. D. 1996, ApJ, 468, 306

Geffert, M., Kümmel, M. W., \& Schmidt, H. 1995, A\&AS, 112, 229
Guillout, P., Sterzik, M. F., Schmitt, J. H. M. M., et al. 1998, A\&A, 337, 113

Gunn, J. E., Griffin, R. F., Griffin, R. E. M., \& Zimmerman, B. A. 1988 , AJ, 96, 198

Heck, A. 1978, Vistas in Astronomy, 22, 221

Høg, E., Fabricius, C., Makarov, V. V., et al. 2000a, A\&A, 355, L27

Høg, E., Fabricius, C., Makarov, V. V., et al. 2000b, A\&A, 357, 367

Johnson, H. 1966, ARA\&A, 4, 190

Jones, B. F. 1970, AJ, 75, 563

Jones, B. F., \& Herbig, G. H. 1979, AJ, 84, 1872

Lindegren, L., Madsen, S., \& Dravins, D. 2000, A\&A, 356, 1119

Makarov, V. V., Odenkirchen, M., \& Urban, S. 2000, A\&A, 358, 923

Makarov, V. V., \& Urban, S. 2000, MNRAS, 312, 289

Micela, G., Sciortino, S., Harnden Jr., F. R., et al. 1999, A\&A, 341,751

McAlister, H. A., Hartkopf, W. I., Hutter, D. J., et al. 1987, AJ, 93, 688

Patience, J., Ghez, A. M., Reid, I. N., et al. 1998, AJ, 115, 1972

Perryman, M. A. C., Brown, A. G. A., Lebreton, Y., et al. 1998, A\&A, 331, 81

Randish, S., Schmitt, J. H. M. M., Prosser, C. F., \& Stauffer, J. R. 1996, A\&A, 305, 785

Reid, N. 1992, MNRAS, 257, 257

Stern, R. A., Schmitt, J. H. M. M., \& Kahabka, P. T. 1995, ApJ, 448, 683

Sterzik, M. F., Alcalá, J. M., Covino, E., \& Petr, M. G. 1999, A\&A, 346, L41

Torra, J., Fernández, D., \& Figueras, F. 2000, A\&A, 359, 822

Urban, S. E., Wycoff, G. L., \& Makarov, V. V. 2000, AJ, 120, 501

Voges, W., Aschenbach, B., Boller, Th., et al. 1999, A\&A, 349, 389

Webb, R. A., Zuckerman, B., Platais, I., et al. 1999, ApJ, 512, L63

Webb, R. A., \& Zuckerman, B. 2000, AAS 195th Meeting, January 2000 